\title{
The Giant Rheumatoid Nodule: A Case Report
}

Sushilkumar Satish Gupta, Ankur Sinha and Vinod Namana*

Maimonides Medical Center, Ny, United States

Corresponding author: Vinod Namana, Cardiovascular Disease Fellow, Maimonides Medical Center, Brooklyn, NY 11219, Tel: 917-445-7267; E-mail: vnamana@maimonidesmed.org

Rec date: May 04, 2017; Acc date: June 5, 2017; Pub date: June 9, 2017

Copyright: (c) 2017 Namana V. This is an open-access article distributed under the terms of the Creative Commons Attribution License, which permits unrestricted use, distribution, and reproduction in any medium, provided the original author and source are credited.

\begin{abstract}
Rheumatoid arthritis is one of the most common debilitating rheumatological disorders. A subcutaneous rheumatoid nodule usually occurs in advanced cases of rheumatoid arthritis and is also a hallmark of the chronicity of the disease. They are also the most common extra-articular manifestation of rheumatoid arthritis. We present a case of a giant rheumatoid nodule, developed on the left elbow.
\end{abstract}

Keywords: Rheumatoid nodule; Pain; Arthritis

\section{Case Report}

An 87-year-old female presented to our hospital for pain and redness in her left foot. She also complained of fever from past couple of days. Her past medical history was significant for chronic obstructive pulmonary disease, rheumatoid arthritis (RA), hypertension, and hypothyroidism. She was diagnosed with acute osteomyelitis based on the magnetic resonance imaging findings, elevated erythrocyte sedimentation rate and C-reactive protein. She was treated with intravenous antibiotics for a period of 6 weeks and discharged from the hospital.

While she was hospitalized, on physical examination, we found an interesting clinical finding. She had severe form of rheumatoid arthritis and rheumatoid nodule on her left elbow joint. As per the patient, she was diagnosed with RA almost 30 years ago, and it was managed with corticosteroids, methotrexate and folic acid supplementation. On examination she had severe ulnar drift of the left hand, subluxation of the second left metacarpal phalangeal joint, Boutonniere deformity (Figure 1) and a very big subcutaneous nodule at the left elbow joint (Figure 2).

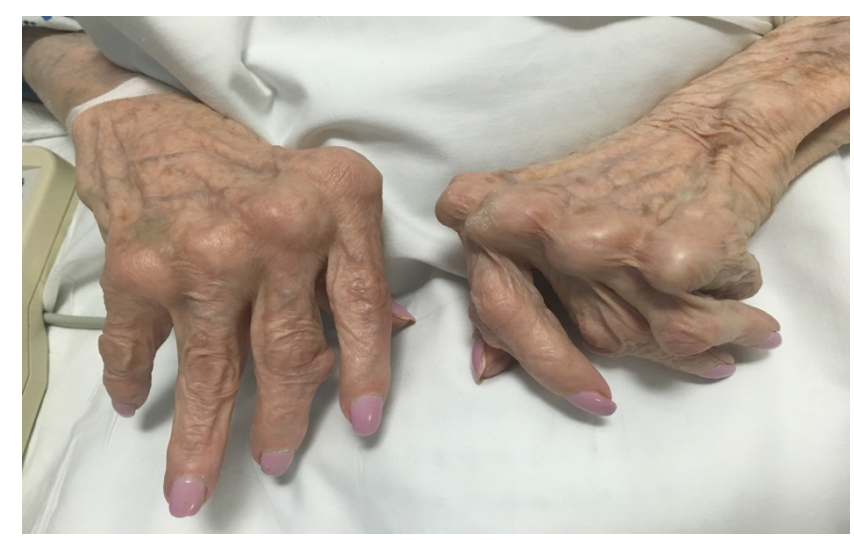

Figure 1: severe joint deformity in Rheumatoid arthritis.
The nodule was pale in color, had doughy consistency, approximately $8 \mathrm{~cm}$ in diameter, and there was no signs of infections or ulcerations. She denied pain at the site of the nodule, but did complain of decreased range of motion. She had tried intra nodular corticosteroid injections in the past without significant relief. During this admission, she did not require any medical treatment for rheumatoid arthritis. The nodule could be mistaken for a gouty tophus, but she never had hyperuricemia or treated for gout. To the best of our knowledge, this is the largest rheumatoid nodule reported ever.

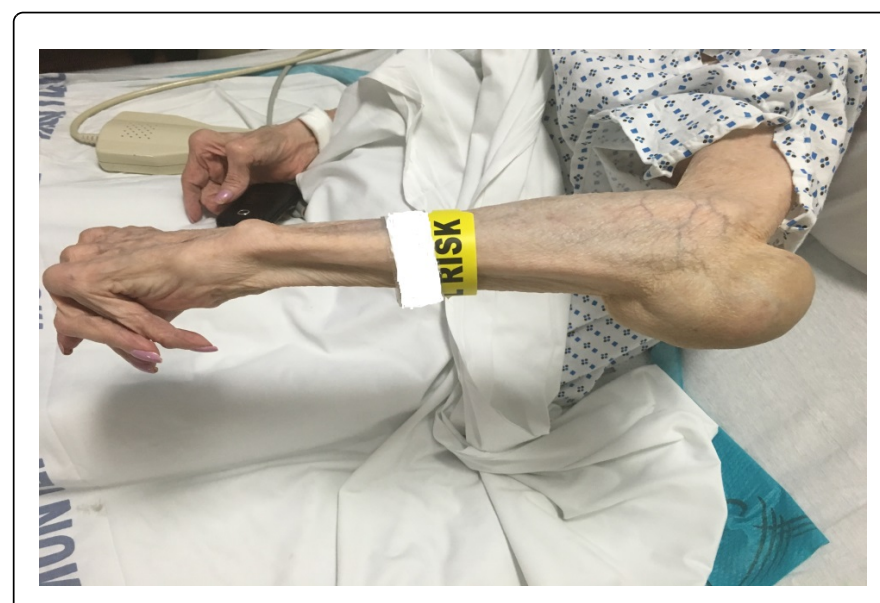

Figure 2: Giant rheumatoid nodule at the left elbow joint.

\section{Discussion}

Rheumatoid nodules (RN) are the most common extra-skeletal manifestation of RA seen in approximately $30 \%$ of the seropositive patients [1]. RN prevalence is highest during the initial stages of disease. $\mathrm{RN}$, when presents along with neutropenia and splenomegaly is known as Felty syndrome, while Caplan syndrome is a combination of RA and coal worker's pneumoconiosis that manifest as intrapulmonary nodule on the chest X-ray. Nodules are commonly seen subcutaneously on the extensor parts of the limbs, but can likewise appear in the visceral organs. Approximately $30 \%$ of autopsy specimens of patients diagnosed with RA are found to have RN in lung 
and cardiac structures [2]. Tissue trauma, elevated rheumatoid factor anti-CCP antibodies and smoking serve as independent risk factors for nodule formation [3-5]. RN is a hallmark of long lasting and active inflammatory state of the disease. The etiology of RN is unclear, and different hypothesis have been postulated in the pathogenesis of $\mathrm{RN}$, however none of them explain why only a portion of patients with RA develop RN. One theory suggests that, RN occur due to repetitive trauma causing local vascular damage and increased levels of proangiogenesis factors, interleukins (IL) that lead to granuloma formation [6]. Other studies believe that localized vasculitis results in complement activation, which in turn leads to vesiculation of endothelial cells, and pooling of rheumatoid factor immune complexes, which eventually leads to accumulation of fibrin [7]. The size usually ranges from several millimeters to 3-4 centimeters. They are normally soft, painless and movable, but can get painful if attached to a bursa or a tendon. Rarely nodules can also develop into an ulcer and increase the risk of secondary bacterial infection. Histopathology of rheumatoid nodules consist of necrobiotic granuloma in the center, surrounded by a palisade of macrophages, fibroblast and $\mathrm{T}$ lymphocytes. The area of central necrosis contains a large population of HLA-DR+staining, which releases IL-1 [8]. Others studies have shown presence of tumor necrosis factor, interferons and IL which create a chronic inflammatory state that leads to nodule formation [9]. Corticosteroids can be used to treat large and painful nodules $[10,11]$; however, patients usually refrain from any intervention as in our case. Surgical procedure is primarily required for the cosmetic purposes, presence of joint immobility due to nodule or if there is a concern for an infection [11].

There is also a separate entity defined as accelerated rheumatoid nodule (ARN), which occurs as a complication of treatment with disease modifying anti-rheumatic drugs (DMARDS) such as methotrexate, etanercept or leflunomide [12]. They are usually small in size, seen on hand, feet or ears, and disappear once the DMARDS are discontinued [12].

\section{Conclusion}

We present a case of the giant rheumatoid nodule and also review the pathophysiology, histology, clinical course and available treatment options.

\section{References}

1. Magro CM, Crowson AN (2003) The spectrum of cutaneous lesions in rheumatoid arthritis. A clinical and pathological study of 43 patients. J Cutan Pathol 30: 1-10.

2. Kitamura A, Matsuno T, Narita M (2004) Rheumatoid arthritis with diffuse pulmonary rheumatoid nodules. Pathol Int 54: 798-802.

3. Shannon TM, Gale ME (1992) Noncardiac manifestations of rheumatoid arthritis in the thorax. J Thorac Imaging 7: 19-29.

4. Mattey DL, Dawes PT, Fisher J (2002) Nodular disease in rheumatoid arthritis: association with cigarette smoking and HLA-DRB1/TNF gene interaction. J Rheumatol 29: 2313-2318.

5. Nyhall-Wahlin BM, Turesson C, Jacobsson LT (2011) The presence of rheumatoid nodules at early rheumatoid arthritis diagnosis is a sign of extra-articular disease and predicts radiographic progression of joint destruction over 5 years. Scand J Rheumatol 40: 81-87.

6. Hodkinson B, Meyer PW, Musenge E, Ally M, Anderson R, et al. (2012) Exaggerated circulating Th-1 cytokine response in early rheumatoid arthritis patients with nodules. Cytokine 60: 561-564.

7. Kato H, Yamakawa M, Ogino T (2000) Complement mediated vascular endothelial injury in rheumatoid nodules: a histopathological and immunohistochemical study. J Rheumatol 27: 1839-1847.

8. Miyasaka N, Sato K, Yamamoto K (1989) Immunological and immunohistochemical analysis of rheumatoid nodules. Ann Rheum Dis 48: 220-226.

9. Stamp LK, Easson A, Lehnigk U (2008) Different T cell subsets in the nodule and synovial membrane: absence of interleukin-17A in rheumatoid nodules. Arthritis Rheum 58: 1601-1608.

10. Ching DW, Petrie JP, Klemp P, Jones JG (1992) Injection therapy of superficial rheumatoid nodules. Br J Rheumatol 31: 775-777.

11. Baan H, Haagsma CJ, van de Laar MA (2006) Corticosteroid injections reduce size of rheumatoid nodules. Clin Rheumatol 25: 21-23.

12. García-Patos V (2007) Rheumatoid nodule. Semin. Cutan. Med. Surg 26: 100-107. 